Pacific Journal of Mathematics

ON THE IDEAL STRUCTURE OF SOME ALGEBRAS OF 


\title{
ON THE IDEAL STRUCTURE OF SOME ALGEBRAS OF ANALYTIC FUNCTIONS
}

\author{
JoHN E. GILBERT
}

\begin{abstract}
Using the Beurling-Lax description of invariant subspaces of $H^{2}(R)$, we describe the ideal structure of two large classes of convolution algebras whose Fourier-Laplace Transforms are entire functions. A closed ideal will be characterized by its cospectrum or by its cospectrum together with a nonnegative number related to the "rate of decrease at infinity"; in the latter case, the closed ideals having the same cospectrum form a totally ordered family $\left\{I_{\xi}\right\}, \xi \in[0, \infty)$, with $I_{\xi} \supsetneqq I_{\eta}$ whenever $\xi<\eta$. New examples of algebras to which the results apply are given.
\end{abstract}

The familiar notation for the spaces considered by Schwartz ([9]) is adopted and each space is equipped with its usual topology. Let $\mathscr{K}$ be the subspace of $\mathscr{E}(R)$ of functions $\phi$ for which

$$
\|\phi\|_{k}=\sup _{x \in R, p \leqq k} \exp (k|x|)\left|D^{p} \phi(x)\right|
$$

is finite for each $k=0,1, \cdots$; the topology on $\mathscr{K}$ will be the one induced by the semi-norms $\|(\cdot)\|_{k}, k=0,1, \ldots$ Under this topology $\mathscr{K}$ is a convolution algebra with separately continuous multiplication. A detailed discussion of $\mathscr{K}$ along with associated spaces is given in [4], [12] and [13] (note that Zielézny uses $\mathscr{K}_{1}$ instead of $\mathscr{K}$ ). We recall some of the results in the form most convenient for applications here.

Denote by $\mathscr{O}_{c}^{\prime}(\mathscr{K})$ the convolution operators on $\mathscr{K}$, i.e., the distributions $S \in \mathscr{D}^{\prime}(R)$ for which the convolution operator $\phi \rightarrow S * \dot{\phi}$ is well-defined and continuous from $\mathscr{K}$ into $\mathscr{K}$. $\mathscr{O}_{c}^{\prime}(\mathscr{K})$ is given the topology it inherits as a subspace of $\mathscr{L}_{b}(\mathscr{K}, \mathscr{K})$, the continuous linear mappings from $\mathscr{K}$ into $\mathscr{K}$, when $\mathscr{L}_{b}(\mathscr{K}, \mathscr{K})$, has the topology of uniform convergence on bounded subsets of $\mathscr{K}$. Alternatively, if $\mathscr{K}^{\prime}$ is the strong dual of $\mathscr{K}, \mathscr{O}_{c}^{\prime}(\mathscr{K})$ can be defined as the space $\mathscr{O}_{c}^{\prime}\left(\mathscr{K}^{\prime}, \mathscr{K}^{\prime}\right)$ of convolution operators on $\mathscr{K}^{\prime}$ in the sense of Schwartz ([10], exposé 10) and given the topology acquired as a subspace of $\mathscr{L}_{b}\left(\mathscr{K}^{\prime}, \mathscr{K}^{\prime}\right)$. These two definitions of $\mathscr{O}_{c}^{\prime}(\mathscr{K})$ are, however, entirely equivalent (cf. [13, Ths. $\left.\left.2\left(d^{\prime}\right), 4\right]\right)$.

THEOREM 1. The space $\mathcal{O}_{c}^{\prime}(\mathscr{K})$ is a convolution algebra for which

(i) $(S, T) \rightarrow S * T$ is a separately continuous mapping from $\mathscr{O}_{c}^{\prime}(\mathscr{K}) \times \mathscr{O}_{c}^{\prime}(\mathscr{K})$ into $\mathscr{O}_{c}^{\prime}(\mathscr{K})$, 
(ii) $(S, \phi) \rightarrow S * \phi$ is a separately continuous mapping from $O_{0}^{\prime}(\mathscr{K}) \times \mathscr{K}$ into $\mathscr{K}$.

Proof. (i ) See [12, p. 319] for instance, or, more directly, use the definition of the $\mathscr{L}_{b}\left(\mathscr{K}^{\prime}, \mathscr{K}^{\prime}\right)$ topology.

(ii) The continuity of $\phi \rightarrow S * \phi$ follows immediately from the definition of $S$ while the continuity of $S \rightarrow S * \phi$ follows from the definition of the $\mathscr{L}_{b}(\mathscr{K}, \mathscr{K})$ topology on $\mathscr{O}_{c}^{\prime}(\mathscr{K})$.

The Fourier-Laplace Transform $\Phi(z)$ of $\phi \in \mathscr{K}$ defined by

$$
\Phi(z)=\hat{\phi}(z)=\int_{-\infty}^{\infty} \phi(x) e^{-x z} d x, \quad z=u+i v,
$$

can be extended to $\mathscr{O}_{c}^{\prime}(\mathscr{K})$ via the Parseval formula in the usual way since $\mathscr{O}_{c}^{\prime}(\mathscr{K}) \subset \mathscr{K}^{\prime}$. For both $\mathscr{K}$ and $\mathscr{O}_{c}^{\prime}(\mathscr{K})$ the corresponding spaces $K, \mathscr{O}_{M}(K)$ of Fourier-Laplace Transforms $\hat{\phi}, \hat{S}$ respectively, are algebras of entire functions under pointwise multiplication; more precisely, if $S_{\alpha}$ denotes the strip $\{z:|R l(z)| \leqq \alpha\}$ in the complex plane:

THEOREM 2. An entire function $\Phi$

(i) belongs to $K$ if and only if for each positive integer $n$

$$
\sup _{z \in S_{n}}(1+|z|)^{n}|\Phi(z)|<\infty,
$$

(ii) belongs to $\mathcal{O}_{M}(K)$ if and only if there corresponds to each positive integer $n$ an integer $l$ for which

$$
\sup _{z \in S_{n}}(1+|z|)^{-l}|\Phi(z)|<\infty \text {. }
$$

Proof. See [4], [13].

These spaces $K, \mathcal{O}_{M}(K)$ are given the topology carried over from $\mathscr{K}, \mathscr{O}_{c}^{\prime}(\mathscr{K})$ respectively by the Fourier-Laplace Transform. Just as $\mathscr{O}_{c}^{\prime}(\mathscr{K})$ is the algebra of convolution operators on $\mathscr{K}$, so $\mathscr{O}_{M}(K)$ is the algebra of multiplication operators on $K$. This is in complete analogy with the spaces $\mathcal{O}_{c}^{\prime}, \mathcal{O}_{M}$ introduced by Schwartz ([9] $]_{\mathrm{II}}$, p. 99) where the space corresponding to $\mathscr{K}$ is then the space $\mathscr{S}$ of indefinitely differentiable functions of rapid decay at infinity (see [12] for elaboration).

Finally, $\mathscr{K}_{+}$(respectively $\left.\mathcal{O}_{c}^{\prime}\left(\mathscr{K}_{+}\right)_{+}\right)$denotes the subspace of functions in $\mathscr{K}$ (respectively distributions in $\mathscr{O}_{c}^{\prime}(\mathscr{K})$ ) with support in $R_{+}=[0, \infty)$.

2. Throughout the paper $\mathscr{A}$ will denote a topological convolution subalgebra of $\mathscr{O}_{c}^{\prime}(\mathscr{K})$ in which the convolution operation is assumed to be separately continuous. We shall further assume that 
$\mathscr{A}$ contains an approximate identity of functions $\left\{\phi_{k}\right\}$ in $\mathscr{K}_{c}$ or $\mathscr{K}_{+}$ in the sense that $S * \phi_{k}$ converges to $S$ in $\mathscr{A}$ for each $S \in \mathscr{A}$. Now associated with each closed ideal $I$ in $\mathscr{A}$ is the cospectrum cosp $(I)$ of $I$ consisting of the zeros counted according to multiplicity common to the Fourier-Laplace Transform of elements in $I$. If, in addition, $\mathscr{A} \subset \mathscr{O}_{c}^{\prime}(\mathscr{K})_{+}$so that $S \in \mathscr{A}$ has support in $[0, \infty), a_{S}$ will denote the largest nonnegative number such that $S$ has support in $\left[a_{s}, \infty\right)$, i.e., the convex support of $S$ lies in $\left[a_{s}, \infty\right)$ but not in $[c, \infty)$ for any $c>a_{s}$. It is known that $a_{s}$ can be characterized as the largest number for which

$$
\left|\exp \left(a_{S} z\right) \hat{S}(z)\right|=O\left(1+|z|^{n}\right),
$$$$
R l(z)>u_{0},
$$

for some integer $n$ and every $u_{0}>0$ (cf. [2, p. 52]). Thus $a_{S}$ is a measure of the rapidity of decay of $\hat{S}$ at infinity. This definition makes equally good sense for any $S \in \mathscr{S}^{\prime}(R)$ with support in $[0, \infty)$.

From the Beurling-Lax theorem describing the invariant subspaces of $H^{2}(R)$ (see [6, p. 165]; [5, p. 107]), we shall deduce the following results ( $\subset$ will always imply continuous embedding):

THEOREM A. Let $\mathscr{A}$ be a topological convolution subalgebra of $\mathcal{O}_{c}^{\prime}(\mathscr{K})$ with

$$
\mathscr{K} \subset \mathscr{A} \subset O_{0}^{\prime}(\mathscr{K})
$$

Then each closed ideal in $\mathscr{A}$ is characterized by its cospectrum.

THEOREM B. Let $\mathscr{A}$ be a topological convolution subalgebra of $\mathscr{O}_{c}^{\prime}(\mathscr{K})_{+}$with

$$
\mathscr{K}_{+} \subset \mathscr{A} \subset \mathcal{O}_{0}^{\prime}(\mathscr{K})_{+} \cdot
$$

Then each closed ideal I in $\mathscr{A}$ is characterized by its cospectrum together with the number

$$
a_{I}=\inf \left\{a_{S}: S \in I\right\} .
$$

For each $\alpha \in R$ denote by $L_{\alpha}^{p}(R), 1 \leqq p<\infty$, the usual (equivalence classes of) functions for which

$$
\|f\|_{p, \alpha}=\left\{\int_{R}(|f(x)| \exp (\alpha|x|))^{p} d x\right\}^{1 / p}
$$

is finite and by $L_{\omega}^{p}$ the intersection $\bigcap_{\alpha \geq 0} L_{\alpha}^{p}(R)$ provided with the topology defined by $\|(\cdot)\|_{p, \alpha}, \alpha \in R_{+}$. Then $L_{\omega}^{p}(R)$ is a convolution subalgebra of $\mathscr{O}_{c}^{\prime}(\mathscr{K})$ satisfying (2) with an approximate identity from $\mathscr{K}$, even from $\mathscr{D}$ (use Theorem 2, for instance). Thus Theorem A applies. Further examples can be obtained by this construction by 
imposing smoothness conditions, say differentiability or suitable Lipschitz conditions, on the functions. In the opposite direction, denote by $W_{\alpha}^{r p}(R)$ the (Sobolev type) space of functions $f$ in $L_{\alpha}^{p}(R)$ with generalized derivatives $D^{j} f$ in $L_{\alpha}^{p}(R), j=1, \cdots, r$, and $W_{\omega}^{r p}(R)$ the intersection $\bigcap_{\alpha \geq 0} W_{\alpha}^{r p}(R)$, both spaces being given the usual topology. Theorem $\mathrm{A}$ applies here also to $W_{\omega}^{r p}(R), r=1,2, \cdots, 1 \leqq p<\infty$. Theorem B applies, for instance, to analogously defined algebras with $R$ replaced by $R_{+}$, extending any function or distribution defined on $R_{+}$to all of $R$ by zero.

3. This section contains preliminary results the first of which reduces the proof of Theorems A, B to the special case when $\mathscr{A}=$ $L_{\omega}^{2}(R), L_{\omega}^{2}\left(R_{+}\right)$respectively.

THEOREm 3. Let $\mathscr{A}$ be a convolution algebra with an approximate identity $\left\{\phi_{k}\right\}$ from $\mathscr{K}_{+}$and satisfying

$$
\mathscr{K}_{+} \subset \mathscr{A} \subset \mathcal{O}_{c}^{\prime}(\mathscr{K})_{+} \cdot
$$

Then there is a one-to-one correspondence between the closed ideals of $\mathscr{A}$ and the closed ideals of $\mathscr{O}_{c}^{\prime}(\mathscr{K})_{+}$. More precisely, every closed ideal $I \subset \mathscr{A}$ is the intersection with $\mathscr{A}$ of a unique closed ideal $J$ in $\mathscr{O}_{c}^{\prime}(\mathscr{K})_{+}$such that

$$
I=J \cap \mathscr{A}, \quad \operatorname{cosp}(I)=\operatorname{cosp}(J), a_{I}=a_{J} ;
$$

conversely, every such intersection $J \cap \mathscr{A}$ is a closed ideal in $\mathscr{A}$ satisfying (5).

REMARK. An entirely analogous result holds when $\mathscr{A}$ contains an approximate identity from $\mathscr{K}$ and satisfies (2).

Proof of Theorem 3. The final assertion is almost obvious in view of (4). On the other hand, if $I$ is a closed ideal in $\mathscr{A}$, certainly there exists at least one closed ideal $J$ in $\mathscr{O}_{c}^{\prime}(\mathscr{K})_{+}$satisfying (5); for let $J$ be the closure of $I$ in $\mathscr{O}_{c}^{\prime}(\mathscr{K})_{+}$. Then, clearly, $I \subset J \cap \mathscr{A}$, $\operatorname{cosp}(I)=\operatorname{cosp}(J)$ and $a_{I}=a_{J}$. Now, when $\left\{f_{n}\right\}$ is a net in $I$ converging in $\mathscr{O}_{c}^{\prime}(\mathscr{K})_{+}$to $g \in J \cap \mathscr{A}$, by Theorem 1 (ii) the net $\left\{f_{n}{ }^{*} \phi_{k}\right\}$ converges for each $k$ to $g^{*} \phi_{k}$ in $\mathscr{K}_{+}^{*}$ and hence in $\mathscr{A}$. But then $g^{*} \phi_{k} \in I$ and so $g$ itself belongs to $I$, i.e., $I \supset J \cap \mathscr{A}$.

To check the uniqueness, suppose $J_{1}, J_{2}$ are closed ideals in $\mathscr{O}_{c}^{\prime}(\mathscr{K})_{+}$ for which $J_{1} \cap \mathscr{A}=I=J_{2} \cap \mathscr{A}$. Now $I$ contains $g * \mathscr{K}_{+}$for each $g \in J_{1}, J_{2}$ so $I$ contains dense subsets of both $J_{1}$ and $J_{2}$ since $\mathcal{O}_{c}^{\prime}(\mathscr{K})_{+}$ has an approximate identity from $\mathscr{K}_{+}$. Hence, with the notation of the previous paragraph, $J_{1}=J=J_{2}$. 
Assuming Theorem B we obtain very easily the characterization mentioned in the introduction of the closed ideals in $\mathscr{A}$ having the same cospectrum.

CoROLLARY. Under the hypotheses of Theorem 3 the closed ideals in $\mathscr{A}$ having the same cospectrum form a totally ordered family $\left\{I_{\xi}\right\}, \xi \in[0, \infty)$, with $I_{\xi} \supsetneq I_{\eta}$ whenever $\xi<\eta$.

Proof. It is enough to prove the result for $\mathscr{A}=\mathscr{O}_{c}^{\prime}(\mathscr{K})_{+}$(cf. (5)). Let $I$ be any closed ideal in $\mathscr{O}_{c}^{\prime}(\mathscr{K})_{+}$. If $a_{I} \neq 0$, say $a_{I}=\lambda$, the set $I_{0}$ of $\lambda$-left translates

$$
I_{0}=\left\{S_{-\lambda}: S \in I, S_{-\lambda}(x)=S(x+\lambda)\right\}
$$

(obvious modifications if $S$ is not a function) is a closed ideal in $\mathcal{O}_{c}^{\prime}(\mathscr{C})_{+}$with $\operatorname{cosp}\left(I_{0}\right)=\operatorname{cosp}(I)$ and $a_{I_{0}}=0$. When $a_{I}=0$ merely set $I_{0}=I$. Now define $I_{\xi}, \xi \in[0, \infty)$ by

$$
I_{\xi}=\left\{S_{\xi}: S \in I_{0}, S_{\xi}(x)=S(x-\xi)\right\},
$$

the $\xi$-right translates of elements in $I_{0}$. This family $\left\{I_{\xi}\right\}, \xi \in[0, \infty)$, of closed ideals in $\mathscr{O}_{c}^{\prime}(\mathscr{K})_{+}$certainly satisfies $\operatorname{cosp}\left(I_{\xi}\right)=I, a_{I_{\xi}}=\xi$ as is easy to see; hence it is totally ordered by reverse inclusion. Of course, the original ideal $I$ is $I_{\lambda}$ in the family. By Theorem B any closed ideal having the same cospectrum as $I$ belongs to $\left\{I_{\xi}\right\}$.

For the strip $S_{\alpha}, H^{2}\left(S_{\alpha}\right)$ denotes the space of functions analytic in the interior of $S_{\alpha}$ for which

$$
\|F\|=\sup _{|u|<\alpha}\left\{\int_{R}|F(u+i v)|^{2} d v\right\}^{1 / 2}
$$

is finite, $\widetilde{H}^{2}\left(S_{\alpha}\right)$ then denotes the space

$$
\widetilde{H}^{2}\left(S_{\alpha}\right)=\left\{G: G=\left(\cos \frac{\pi z}{4 \alpha}\right) F, F \in H^{2}\left(S_{\alpha}\right)\right\} .
$$

It is well known that $L_{\alpha}^{2}(R)$ is isomorphic to $H^{2}\left(S_{\alpha}\right)$ under the FourierLaplace Transform (cf. [11, p. 130]). On the other hand, $\widetilde{H}^{2}\left(S_{\alpha}\right)$ consists of those functions $L^{2}$-integrable on the boundary $\partial S_{\alpha}$ of $S_{\alpha}$ with respect to the measure $(\cosh (\pi v / 2 \alpha))^{-1} d v$ whose Poisson integrals are analytic in the interior of $S_{\alpha}$. This can be checked by considering for instance the mapping $\zeta \rightarrow z=(4 \alpha / \pi) \tan ^{-1} i \zeta$ of the closed unit disc onto $S_{\alpha}$. When $\widetilde{H}^{2}\left(S_{\alpha}\right)$ is given the norm

$$
\|G\|=\left\{\int_{\partial S_{\alpha}}|G( \pm \alpha+i v)|^{2}\left(\cosh \frac{\pi v}{2 \alpha}\right)^{-1} d v\right\}^{1 / 2},
$$

it is easy to see the mapping $z \rightarrow w=\exp (i \pi z / 2 \alpha)$ of $S_{\alpha}$ onto the 
right hand half-plane $R l(w) \geqq 0$ induces an isomorphism between $\widetilde{H}^{2}(R)$ (cf. [5, p. 107]) $)^{1}$ and $\widetilde{H}^{2}\left(S_{\alpha}\right)$. Since $\widetilde{H}^{2}(R)$ is isomorphic with the usual $H^{2}$ space for the unit disc $([5, \mathrm{p} .105])$ the significance of $\widetilde{H}^{2}\left(S_{\alpha}\right)$ is not surprising.

The spaces $H^{\infty}\left(S_{\alpha}\right), H^{\infty}(R)$ of functions bounded and analytic in the strip $S_{\alpha}$ and the right half-plane respectively are isometrically isomorphic under the mapping $z \rightarrow \exp (i \pi z / 2 \alpha)$. Thus, each $F \in H^{\infty}\left(S_{\alpha}\right)$ admits a factorization in the form

$$
F(z)=\lambda \exp \left(-\rho_{-} e^{i \pi z / 2 \alpha}-\rho_{+} e^{-i \pi z / 2 \alpha}\right) F_{I}(z) F_{0}(z)
$$

with $|\lambda|=1, \rho_{-}$and $\rho_{+}$in $R_{+}, F_{I}$ an "inner" function and $F_{0}$ an "outer" function by transferring the usual factorization for $H^{\infty}(R)$ to $H^{\infty}\left(S_{\alpha}\right)$ (cf. [5, p. 133]). Each "inner" function can be further decomposed again by transferring the analogous decomposition for the half-plane case; at the risk of confusion the same terminology is used as in the half-plane case-Blaschke product, $\cdots$.

We shall denote by $H_{+}^{2}\left(S_{\alpha}\right)$ the closed subspace of $H^{2}\left(S_{\alpha}\right)$ corresponding under the Fourier-Laplace Transform to the closed subspace $L_{\alpha}^{2}\left(R_{+}\right)$of $L_{\alpha}^{2}(R)$. A doubly-invariant subspace $I$ of $H^{2}\left(S_{\alpha}\right)$ will mean one invariant under multiplication by $e^{a z}, a \in R$, a simply invariant subspace of $H_{+}^{2}\left(S_{\alpha}^{\prime}\right)$ one invariant under multiplication by $e^{-a z}, a \in R_{+}$.

THEOREM 4. (a) Each closed doubly-invariant subspace $I$ of $H^{2}\left(S_{\alpha}\right)$ is of the form $I=F H^{2}\left(S_{\alpha}\right)$ for some inner function $F \in H^{\infty}\left(S_{\alpha}\right)$.

(b) If $I$ is a closed simply-invariant subspace of $H_{+}^{2}\left(S_{\alpha}\right)$ then

$$
I=e^{-\rho z} G H_{+}^{2}\left(S_{\alpha}\right)
$$

for some $\rho \in R_{+}$and $G$ a function bounded and analytic in $R l(z)>$ $-\alpha$ having measurable boundary values of modules 1 a.e. on $R l(z)=$ $-\alpha$.

A simple lemma is needed in the proof of Theorem 4 .

LEMma 1. A closed doubly-invariant subspace $I$ of $H^{2}\left(S_{\alpha}\right)$ is invariant under multiplication by every $\Psi \in H^{\infty}\left(S_{\alpha}\right)$.

Proof. The subspace $J$ of $L_{\alpha}^{2}(R)$ corresponding to $I$ is invariant under translation both to the left and to the right. Now, by Plancherel's theorem, the mapping $F \rightarrow \Psi F$ for $F \in H^{2}\left(S_{\alpha}\right)$ gives rise to a mapping $f \rightarrow f_{\psi}$ of $L_{\alpha}^{2}(R)$ commuting with translation. To prove the lemma therefore, it is enough to show that whenever $\phi \in L_{-\alpha}^{2}(R)$ and $\phi * f^{*}=0$ for all $f \in J$, then $\phi *\left(f_{\psi}\right)^{*}=0$ the convolution $\phi * g^{*}$ be-

\footnotetext{
${ }_{1} \widetilde{H}^{2}(R)=\left\{(1+w) f: f \in H^{2}(R), H^{2}(R)\right.$ the Hardy space for the right half-plane $\}$.
} 
ing defined by

$$
\dot{\phi} * g^{*}(x)=\int_{R} \phi(x+y) g(y) d y \text {. }
$$

But, if $h \in L_{\alpha}^{1}(R) \cap L_{\alpha}^{2}(R)$,

$$
\left(\phi * f_{\psi:}^{*}\right) * h^{*}=\phi *\left(f_{\psi} * h\right)^{*}=\left(\phi * f^{*}\right) * h_{\psi}^{*}=0
$$

as an easy calculation shows. Such functions $h$ are dense in $L_{\alpha}^{2}(R)$ so $\phi * f_{\psi}^{*}=0$.

Proof of Theorem 4. (a) Since $|\cos (\pi z / 4 \alpha)|^{2}=\frac{1}{2} \cosh (\pi v / 2 \alpha)$ on $\partial S_{\alpha}$ the set $\widetilde{I}=(\cos (\pi z / 4 \alpha)) I$ is a closed subspace of $\widetilde{H}^{2}\left(S_{\alpha}\right)$ invariant under multiplication by every $\Psi \in H^{\infty}\left(S_{\alpha}\right)$. Thus the subspace of $\widetilde{H}^{2}(R)$ corresponding to $\widetilde{I}$ under the isomorphism of $\widetilde{H}^{2}\left(S_{\alpha}\right)$ and $\widetilde{H}^{2}(R)$ is of the form $F_{1} \widetilde{H}^{2}(R)$ for some inner function $F_{1} \in H^{\infty}(R)$ applying the Beurling-Lax result (cf. [5, p. 107]). Consequently, for some inner function $F \in H^{\infty}\left(S_{\alpha}\right)$,

$$
\left(\cos \frac{\pi z}{4 \alpha}\right) I=F\left(\cos \frac{\pi z}{4 \alpha}\right) H^{2}\left(S_{\alpha}\right) .
$$

Since $\cos (\pi z / 4 \alpha)$ is zero-free throughout $S_{\alpha}$ the result follows.

(b) Under the mapping $F \rightarrow F_{\alpha}, \quad F_{\alpha}(z)=F(z-\alpha), \quad R l(z) \geqq 0$, $H_{+}^{2}\left(S_{\alpha}\right)$ is isomorphic with $H^{2}(R)$. In addition, the image of any closed simply invariant subspace $I$ of $H_{+}^{2}\left(S_{\alpha}\right)$ is an invariant subspace of $H^{2}(R)$ in the terminology of Hoffman ([5, p. 106]). The expression (7) now follows from the result of $\operatorname{Lax}([6] ;[5, \mathrm{p} .107])$.

As mentioned earlier, if $F$ is the Fourier-Laplace Transform of a distribution in $\mathscr{S}^{\prime}(R)$ with support in $[0, \infty)$, the mapping $F \rightarrow a_{F}$ with $a_{F}$ the largest number for which (1) holds, is well-defined. This applies in particular to functions in $H^{2}(R)$ or $H^{\infty}(R)$.

THEOREM 5. If $F=\lambda e^{-\rho z} F_{1} F_{0}$ is the usual factorization of a function $F \in H^{2}(R)$ or $H^{\infty}(R)$, then $\rho=a_{F}$.

THEOREM 6. When $F \in H^{\infty}\left(S_{\alpha}\right)$ is factorized in the form (6) the numbers $\rho_{+}, \rho_{-}$satisfy

$$
\begin{aligned}
& \lim _{v \rightarrow-\infty} \frac{\log |F(u+i v)|}{\exp \left(-\frac{\pi v}{2 \alpha}\right)}=-\rho_{-} \cos \frac{\pi u}{2 \alpha} \\
& \lim _{v \rightarrow \infty} \frac{\log |F(u+i v)|}{\exp \left(\frac{\pi v}{2 \alpha}\right)}=-\rho_{+} \cos \frac{\pi u}{2 \alpha}
\end{aligned}
$$


for almost all $u,|u|<\alpha$. In particular, if $F$ belongs also to $H^{\infty}\left(S_{\beta}\right)$ for some $\beta>\alpha$, then $\rho_{+}=\rho_{-}=0$.

A proof of Theorem 5 appears, for instance, in [8, Lemma 4]. Actually, the Ahlfors-Heins theorem [1, Th. A] gives an even stronger result since

$$
\lim _{r \rightarrow \infty} \frac{\log \left|F\left(r e^{i \theta}\right)\right|}{r}=-\rho \cos \theta
$$

for almost all $\theta,-\pi / 2<\theta<\pi / 2 .^{2}$ To prove Theorem 6 it is enough to establish the first of the limits since the second follows after a transformation $z \rightarrow \bar{z}$. But, when $S_{\alpha}$ is mapped onto $R l(w) \geqq 0$ via the mapping $z \rightarrow w=\exp (i \pi z / 2 \alpha)$, the limit (8) is precisely the analogue for the strip $S_{\alpha}$ of (9). Finally, when $\rho_{-}, \rho_{-}^{\prime}$ are corresponding numbers in the factorization of $F$ as a function in $H^{\infty}\left(S_{\alpha}\right), H^{\infty}\left(S_{\beta}\right)$ respectively, we deduce

$$
\lim _{v \rightarrow-\infty} \frac{\log |F(u+i v)|}{\exp \left(-\frac{\pi v}{2 \beta}\right)}=-\rho_{-}^{\prime} \cos \frac{\pi u}{2 \beta},
$$

for almost all $u,|u|<\beta$, in addition to (8). Choosing any $u,|u|<$ $\alpha$, on which (8) and (10) hold simultaneously we can soon check that $\rho_{-}$must be zero if $\beta>\alpha$. Similarly $\rho_{+}=0$.

4. The proofs of Theorems A and B can now be given.

Proof of A. In view of the remark following Theorem 3, Theorem A need be proved only in the case $\mathscr{A}=L_{\omega}^{2}(R)$.

Let $I$ be a closed ideal in $L_{\omega}^{2}(R), I_{\alpha}$ the closure of $I$ in $L_{\alpha}^{2}(R)$. Then $I=\bigcap_{\alpha>0} I_{\alpha}$. For certainly $I \subset \bigcap_{\alpha \geq 0} I_{\alpha}$; on the other hand, the topology on $L_{\omega}^{2}(R)$ being the topology defined by the semi-norms $\|(\cdot)\|_{\alpha}$, i.e., the projective limit topology, each $f \in \bigcap_{\alpha \geqq 0} I_{\alpha}$ is a limit point of $I$ in $L_{\omega}^{2}(R)$ hence $\bigcap_{\alpha \geqq 0} I_{\alpha}=I$. The set $J_{\alpha}$ of Fourier Laplace Transforms of functions in $I_{\alpha}$ is a closed doubly-invariant subspace of $H^{2}\left(S_{\alpha}\right)$. Thus $J_{\alpha}=F H^{2}\left(S_{\alpha}\right)$ where $F$ is an inner function in $H^{\infty}\left(S_{\alpha}\right)$ depending on $\alpha$ of course. In the factorization of $F$

$$
F=\exp \left(-\rho_{-} e^{i \pi z / 2 \alpha}-\rho_{+} e^{-i \pi z / 2 \alpha}\right) B S,
$$

with $B$ a Blaschke product, $S$ a singular function, the Blaschke product is formed with the elements of $\operatorname{cosp}(I)$ lying in $S_{\alpha} \mid \partial S_{\alpha}$. On the

${ }^{2}$ In the application of (9) we have in mind the singular function in $F$ is identically 1. A proof of (9) in this case avoiding the Ahlfors-Heins theorem is given in [7] (for the upper half-plane) on page 243. 
other hand, if $\alpha$ is chosen so that $\partial S_{\alpha}$ does not intersect $\operatorname{cosp}(I)$, the singular function in (11) is identically 1 ; for if $z_{0} \in \partial S_{\alpha}$, there exists $f \in I$ with $\hat{f}$ continuous on $\partial S_{\alpha}$ and nonzero at $z_{0}$ in which case $z_{0}$ does not belong to the support of the singular measure defining $S$ (cf. [5, p. 70]). Furthermore, as each $\hat{f}, f \in I$, belongs to $H^{\infty}\left(S_{\beta}\right)$ for every $\beta>\alpha$, the constants $\rho_{+}, \rho_{-}$in the factorization of $\hat{f}$, and hence in (11), are both zero. Thus, with this choice of $\alpha$, the inner function reduces to the Blaschke product formed by the elements of $\operatorname{cosp}(I)$ in $S_{\alpha}$.

Now choose a monotonic unbounded sequence of $\alpha$ 's for which $\operatorname{cosp}(I) \cap \partial S_{\alpha}$ is empty. Such a choice is always possible since any such sequence is enough to describe $L_{\omega}^{2}(R)$ both algebraically and topologically. If $f$ is any function in $L_{\omega}^{2}(R)$ for which $\hat{f}(z)=0$ whenever $z \in \operatorname{cosp}(I)$ (with appropriate multiplicities), it is clear that $\hat{f}$ belongs to every $J_{\alpha}$ because the corresponding inner function (11), merely a Blaschke product, divides $\hat{f}$. Consequently, $f \in \bigcap_{\alpha \geqq 0} I_{\alpha}=I$ showing that $I$ is determined by $\operatorname{cosp}(I)$.

Proof of B. In this case it is enough to consider $L_{\omega}^{2}\left(R_{+}\right)$. For a closed ideal $I$ in $L_{\omega}^{2}\left(R_{+}\right)$, let $I_{\alpha}$ be its closure in $L_{\alpha}^{2}\left(R_{+}\right)$. By the same argument as in the proof of $A$ we have $I=\bigcap_{\alpha \geqq 0} I_{\alpha}$. The corresponding set $J_{\alpha}$ of Fourier-Laplace Transforms is a simply invariant subspace of $H_{+}^{2}\left(S_{\alpha}\right)$ so is given by

$$
J_{\alpha}=e^{-\rho z} G H_{+}^{2}\left(S_{\alpha}\right)
$$

for some $\rho \in R_{+}$and "inner" function $G$. By much the same argument as in the proof of Theorem A, if $\alpha$ belongs to a suitably chosen sequence, $G$ consists only of the Blaschke product for a half-plane formed with the elements of $\operatorname{cosp}(I)$ in the half-plane $R l(z)>-\alpha$. Also, by Theorem 5, the number $\rho$ in (12) is given by

$$
\rho=\inf \left\{a_{F}: F \in J_{\alpha}\right\}
$$

since $e^{-\rho z} G$ is the greatest common divisor of the inner functions in the factorization of elements in $J_{\alpha}$. But then, with the notation of (3), $\rho=a_{I}$. For certainly $\rho \leqq a_{I}$ since $I_{\alpha} \supset I$; on the other hand, the limit in $L_{\alpha}^{2}\left(R^{+}\right)$of any sequence with convex support in $\left[a_{I}, \infty\right)$ again has convex support in $\left[a_{I}, \infty\right)$-hence $\rho=a_{I}$. Thus any $f \in L_{\omega}^{2}\left(R_{+}\right)$ which is zero a.e. outside $\left[\alpha_{I}, \infty\right)$ and whose Fourier-Laplace Transform $\hat{f}$ is zero on $\operatorname{cosp}(I)$ (with appropriate multiplicities), belongs to each $I_{\alpha}$, hence to $I=\bigcap_{\alpha \geq 0} I_{\alpha}$. Thus $I$ is determined by $\operatorname{cosp}(I)$ together with the number $a_{I}$. 


\section{REFERENCES}

1. L. Ahlfors and M. Heins, Questions of regularity connected with the PhragmenLindelöf principle, Ann. of Math. (2) 50 (1949), 341-346.

2. E. Beltrami and M. Wohlers, Distributions and the Boundary Values of Analytic Functions, Academic Press, New York, 1966.

3. R. P. Boas, Entire Functions, Academic Press, New York, 1954.

4. M. Hasumi, Note on the n-dimensional tempered ultra-distributions, Tôhoku Math.

J. 13 (1961), 94-104.

5. K. Hoffman, Banach Spaces of Analytic Functions, Prentice-Hall, Englewood Cliffs, N. J., 1962.

6. P. Lax, Translation invariant subspaces, Acta Math. 101 (1959), 163-178.

7. B. Ja. Levin, Distribution of Zeros of Entire Functions, Amer. Math. Soc. Trans. of Math. Monographs, vol. 5, Providence, R. I., 1964.

8. B. Nyman, On the one-dimensional translation group and semi-group in certain function spaces, Thesis, Uppsala, 1950.

9. L. Schwartz, Théorie des Distributions, vols. I, II, Hermann, Paris, 1957, 1959.

10. - Séminaire Schwartz, Paris, 1953-1954.

11. E. C. Titchmarsh, Introduction to the Theory of Fourier Integrals, Oxford Univ. Press, Oxford, 1948.

12. Z. Zielézny, Hypoelliptic and entire elliptic convolution equations in subspaces of the space of distributions (I), Studia Math. 28 (1967), 317-332.

13. On the space of convolution operators in $\mathscr{K}^{\prime}{ }^{\prime}$, Studia Math. 31 (1968), $111-124$.

Received September 30, 1969, and in revised form April 25, 1970. 


\section{PACIFIC JOURNAL OF MATHEMATICS}

\section{EDITORS}

\author{
H. SAMELSON \\ Stanford University \\ Stanford, California 94305 \\ Richard Pierce \\ University of Washington \\ Seattle, Washington 98105
}

\author{
J. DugundJI \\ Department of Mathematics \\ University of Southern California \\ Los Angeles, California 90007 \\ RICHARD ARENS \\ University of California \\ Los Angeles, California 9.0024
}

\section{ASSOCIATE EDITORS}

\begin{tabular}{|c|c|}
\hline E. F. BECKENBACH & K. YoshidA \\
\hline \multicolumn{2}{|c|}{ SUPPORTING INSTITUTIONS } \\
\hline UNIVERSITY OF BRITISH COLUMBIA & STANFORD UNIVERSITY \\
\hline CALIFORNIA INSTITUTE OF TECHNOLOGY & UNIVERSITY OF TOKYO \\
\hline UNIVERSITY OF CALIFORNIA & UNIVERSITY OF UTAH \\
\hline MONTANA STATE UNIVERSITY & WASHINGTON STATE UNIVERSITY \\
\hline UNIVERSITY OF NEVADA & UNIVERSITY OF WASHINGTON \\
\hline NEW MEXICO STATE UNIVERSITY & $* \quad * \quad *$ \\
\hline OREGON STATE UNIVERSITY & AMERICAN MATHEMATICAL SOCIETY \\
\hline UNIVERSITY OF OREGON & CHEVRON RESEARCH CORPORATION \\
\hline OSAKA UNIVERSITY & TRW SYSTEMS \\
\hline UNIVERSITY OF SOUTHERN CALIFORNIA & NAVAL WEAPONS CENTER \\
\hline
\end{tabular}

The Supporting Institutions listed above contribute to the cost of publication of this Journal, but they are not owners or publishers and have no responsibility for its content or policies.

Mathematical papers intended for publication in the Pacific Journal of Mathematics should be in typed form or offset-reproduced, (not dittoed), double spaced with large margins. Underline Greek letters in red, German in green, and script in blue. The first paragraph or two must be capable of being used separately as a synopsis of the entire paper. The editorial "we" must not be used in the synopsis, and items of the bibliography should not be cited there unless absolutely necessary, in which case they must be identified by author and Journal, rather than by item number. Manuscripts, in duplicate if possible, may be sent to any one of the four editors. Please classify according to the scheme of Math. Rev. Index to Vol. 39. All other communications to the editors should be addressed to the managing editor, Richard Arens, University of California, Los Angeles, California, 90024.

50 reprints are provided free for each article; additional copies may be obtained at cost in multiples of 50 .

The Pacific Journal of Mathematics is published monthly. Effective with Volume 16 the price per volume (3 numbers) is $\$ 8.00$; single issues, $\$ 3.00$. Special price for current issues to individual faculty members of supporting institutions and to individual members of the American Mathematical Society: $\$ 4.00$ per volume; single issues $\$ 1.50$. Back numbers are available.

Subscriptions, orders for back numbers, and changes of address should be sent to Pacific Journal of Mathematics, 103 Highland Boulevard, Berkeley, California, 94708.

PUBLISHED BY PACIFIC JOURNAL OF MATHEMATICS, A NON-PROFIT CORPORATION

Printed at Kokusai Bunken Insatsusha (Internatıonal Academic Printing Co., Ltd.), 7-17, Fujimi 2-chome, Chiyoda-ku, Tokyo, Japan. 


\section{Pacific Journal of Mathematics}

\section{Vol. 35, No. $3 \quad$ November, 1970}

John D. Arrison and Michael Rich, On nearly commutative degree one algebras . . . 533

Bruce Alan Barnes, Algebras with minimal left ideals which are Hilbert spaces . . . . 537

Robert F. Brown, An elementary proof of the uniqueness of the fixed point index . . . 549

Ronn L. Carpenter, Principal ideals in F-algebras .................... 559

Chen Chung Chang and Yiannis (John) Nicolas Moschovakis, The Suslin-Kleene

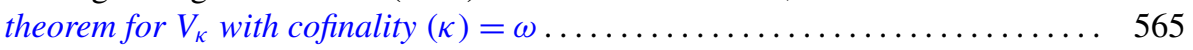

Theodore Seio Chihara, The derived set of the spectrum of a distribution

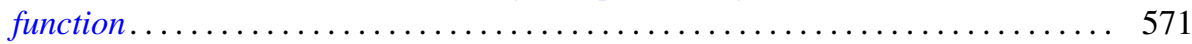

Tae Geun Cho, On the Choquet boundary for a nonclosed subspace of $C(S) \ldots \ldots \quad 575$

Richard Brian Darst, The Lebesgue decomposition, Radon-Nikodym derivative,

conditional expectation, and martingale convergence for lattices of sets .......

David E. Fields, Dimension theory in power series rings . . . . . . . . . . . .

Michael Lawrence Fredman, Congruence formulas obtained by counting

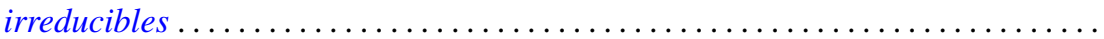

John Eric Gilbert, On the ideal structure of some algebras of analytic functions.....

G. Goss and Giovanni Viglino, Some topological properties weaker than

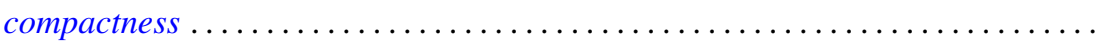

581

601

625

George Grätzer and J. Sichler, On the endomorphism semigroup (and category) of

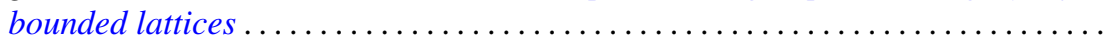

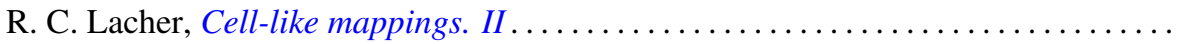

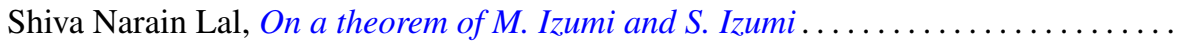

661

Howard Barrow Lambert, Differential mappings on a vector space ...............

Richard G. Levin and Takayuki Tamura, Notes on commutative power joined

semigroups.

Robert Edward Lewand and Kevin Mor McCrimmon, Macdonald's theorem for quadratic Jordan algebras.

J. A. Marti, On some types of completeness in topological vector spaces ....

Walter J. Meyer, Characterization of the Steiner point

717

Saad H. Mohamed, Rings whose homomorphic images are $q$-rings ...

727

Thomas V. O'Brien and William Lawrence Reddy, Each compact orientable surface

of positive genus admits an expansive homeomorphism ...

737

Robert James Plemmons and M. T. West, On the semigroup of binary relations...

743

Calvin R. Putnam, Unbounded inverses of hyponormal operator . .

755

William T. Reid, Some remarks on special disconjugacy criteria for differential

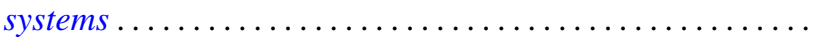

C. Ambrose Rogers, The convex generation of convex Borel sets in euclidean

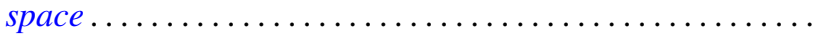

S. Saran, A general theorem for bilinear generating functions .

S. W. Smith, Cone relationships of biorthogonal systems ......

Wolmer Vasconcelos, On commutative endomorphism rings ....

795

Vernon Emil Zander, Products of finitely additive set functions from Orlicz

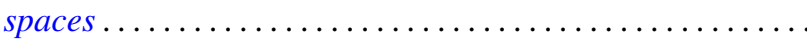

G. Sankaranarayanan and C. Suyambulingom, Correction to: "Some renewal

theorems concerning a sequence of correlated random variables" .

Joseph Zaks, Correction to: "Trivially extending decompositions of $E^{n}$ ”....... 805

Dong Hoon Lee, Correction to: "The adjoint group of Lie groups" ............ 805

James Edward Ward, Correction to: "Two-groups and Jordan algebras". 